\title{
Endotrophin, a multifaceted player in metabolic dysregulation and cancer progression, is a predictive biomarker for the response to PPAR $\gamma$ agonist treatment
}

\author{
Kai Sun ${ }^{1,2}$ • Jiyoung Park $^{1,3}$ • Min Kim ${ }^{1,4,5}$ - Philipp E. Scherer ${ }^{1,6}$
}

Received: 7 September 2016 / Accepted: 13 September 2016 / Published online: 7 October 2016

(C) Springer-Verlag Berlin Heidelberg 2016

\begin{abstract}
Endotrophin is a cleavage product derived from the collagen $\mathrm{VI}(\alpha 3)$ chain. Collagen VI is expressed in a number of different tissues, but adipose tissue is a particularly prominent source for this extracellular matrix constituent. Mice lacking collagen VI are metabolically healthier due to reduced fibrosis in adipose tissue. Endotrophin seems to be one of the key players of collagen VI-mediated signalling effects, including its pro-fibrotic nature and chemoattractant properties for macrophages, while also playing an important role in cancer progression and the chemoresistance of tumour cells. The glucose-lowering class of thiazolidinediones (TZDs) that mediate their action through the nuclear receptor peroxisome proliferator-activated receptor (PPAR) $\gamma$ also exerts important effects on endotrophin by reducing the transcription of
\end{abstract}

Kai Sun, Jiyoung Park and Min Kim contributed equally to this commentary.

Philipp E. Scherer

Philipp.Scherer@utsouthwestern.edu

1 Touchstone Diabetes Center, Department of Internal Medicine, University of Texas Southwestern Medical Center, 5323 Harry Hines Boulevard, Dallas, TX 75390-8549, USA

2 Center for Metabolic and Degenerative Diseases, The Brown Foundation Institute of Molecular Medicine, University of Texas Health Science Center at Houston, Houston, TX, USA

3 Department of Biological Sciences, School of Life Sciences, Ulsan National Institute of Science and Technology, Ulsan, South Korea

4 National Research Laboratory for Mitochondrial Signaling, Department of Physiology, College of Medicine, Inje University, Busan, South Korea

5 Cardiovascular and Metabolic Disease Center, Inje University, Busan, South Korea

6 Department of Cell Biology, University of Texas Southwestern Medical Center, Dallas, TX, USA parental collagen VI molecules. As with many other pharmacological interventions, there is a range of responses observed in a diabetic patient population. In this issue of Diabetologia, Karsdal and colleagues (DOI: 10.1007/s00125-016-4094-1) demonstrate that baseline endotrophin levels offer excellent predictive values to indicate individuals who will show an optimised response to TZDs with respect to the lowering of $\mathrm{HbA}_{1 \mathrm{c}}$ and reduced risk of adverse side effects. The identification of a predictive biomarker for optimal responders is an important step in highlighting the continued viability of TZDs as an effective glucose-lowering class of compounds.

Keywords Adverse events · Biomarker · Fibrosis · Non-responders · PPAR $\gamma$ agonist

$\begin{array}{ll}\text { Abbreviations } \\ \text { ECM } & \text { Extracellular matrix } \\ \text { EMT } & \text { Epithelial-mesenchymal transition } \\ \text { HCC } & \text { Hepatocellular carcinoma } \\ \text { NAFLD } & \text { Non-alcoholic fatty liver disease } \\ \text { NASH } & \text { Non-alcoholic steatohepatitis } \\ \text { PPAR } & \text { Peroxisome proliferator-activated receptor } \\ \text { SAE } & \text { Severe adverse effect } \\ \text { TZD } & \text { Thiazolidinediones }\end{array}$

\section{Introduction}

The peroxisome proliferator-activated receptors (PPARs) are nuclear hormone receptors. There are three subtypes referred to as PPAR $\alpha, \operatorname{PPAR} \beta / \delta$ and PPAR $\gamma$. They exert their main roles as transcription factors, regulating cell development, differentiation and metabolic homeostasis. Specifically, PPAR $\gamma$ 
is widely expressed, but best known as a master regulator of adipocyte differentiation and most abundantly present in adipose tissue [1]. Endogenous ligands for PPAR $\gamma$ have been reported [2], but the most widely known ligands are the synthetic thiazolidinediones (TZDs). TZDs are effective PPAR $\gamma$ agonists [3] and were extensively used for the treatment of type 2 diabetes. Their recent use has been somewhat more limited because of some purported side effects [4]. TZDs improve insulin sensitivity by attenuation of lipolysis [5], thereby reducing plasma NEFA levels [6], reducing oxidative stress [7] and inflammation [8], and increasing adiponectin [9] and fatty acid uptake [10]. They also promote an overall healthy expansion of the subcutaneous adipose tissue that serves as an effective lipid sink. Although many effects of TZDs are mediated by PPAR $\gamma$, there is reason to believe that TZDs can also exert functions independent of PPAR $\gamma$ [11].

\section{TZD responses vary widely amongst different individuals}

Over two decades, TZDs have been extensively studied with regards to their targeted responses, as well as unwanted side effects. Furthermore, there is significant variability in the clinical responses to TZDs. Little is known about this variability in the clinical response to TZDs and what mechanisms are responsible for the differential outcomes. On average, 30\% of diabetic patients do not respond properly to treatment with TZDs; in these individuals, these drugs fail to lower $\mathrm{HbA}_{1 \mathrm{c}}$ and fasting glucose levels. Suter et al demonstrated that troglitazone can improve insulin resistance with a response rate of $73 \%$, although only a small group was studied [12]. According to Igarashi et al, eight out of 23 participants were non-responsive to pioglitazone treatment. They found that BMI, immunoreactive insulin and HOMA-IR indices were markedly higher in the pioglitazone-responsive group compared with non-responders. In addition, atherogenic factors, such as LDL-cholesterol and remnant like particle cholesterol (RLP-C) levels, were higher in responders [13]. Similarly, the response rate was only $57 \%$ in 70 Japanese individuals with type 2 diabetes, as observed by Satoh and colleagues. These authors suggested that adiponectin could be a factor which determines the TZD response [14], consistent with data obtained in rodents [15]. Sears et al reported that $25 \%$ of insulin resistant patients were TZD non-responders. Non-responders exhibited blunted insulin-induced expression of hexokinase 2 (HK2) and pyruvate dehydrogenase kinase 4 (PDK4) [16]. What we conclude from these studies is that there is a great need to determine the individuals who may respond vs those who may not respond to TZDs to prevent unnecessary exposure to the drug, avoiding excess costs and potential sideeffects in patients without any health benefits.

\section{The role of endotrophin in metabolism}

Collagen VI is one of the major extracellular matrix (ECM) proteins secreted from adipocytes. It is composed of three major polypeptide chains, $\alpha 1, \alpha 2$, and $\alpha 3$, which are encoded by distinct genes (COL6A1, COL6A2, and COL6A3, respectively). Additionally, three collagen VI subunits, $\alpha 4, \alpha 5$, and $\alpha 6$ chains, have been identified based on sequence homology with the $\alpha 3$ chain $[17,18]$. Each chain consists of a short collagenous domain linked by a variable number of von Willebrand factor type A (vWF-A) domains. In particular, the longest collagen VI polypeptide chain (the $\alpha 3$ subunit) can release its most C-terminal domain (C5) immediately after the secretion of microfilament tetramers to the extracellular surface. Interestingly, our recent findings indicate that this soluble cleavage product of the collagen $\operatorname{VI}(\alpha 3)$ chain, now referred to as 'endotrophin', is actively involved in various biological processes, such as inflammation, angiogenesis, fibrosis and epithelial-mesenchymal transition (EMT) in the context of cancer (Fig. 1). It also seems to play a critical role in obesity-induced systemic insulin resistance by increasing chronic inflammation and fibrosis in adipose tissues [19-21].

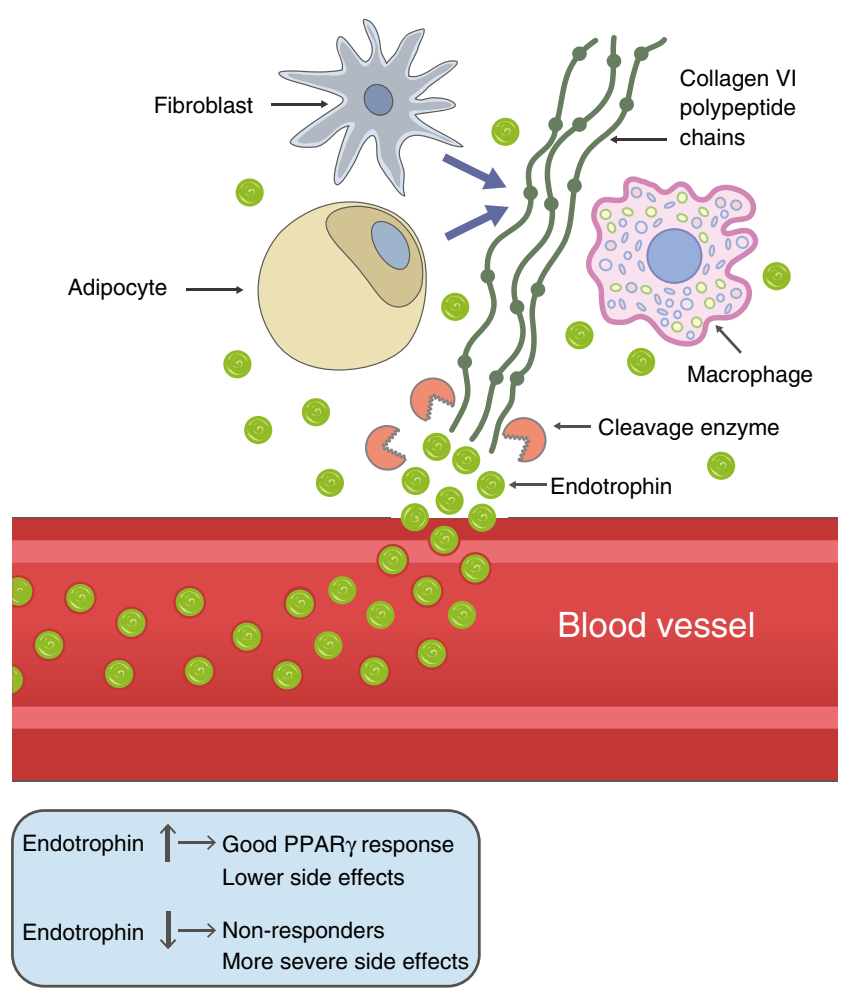

Fig. 1 Endotrophin is a collagen VI-derived cleavage product that originates in adipose tissue, secreted from adipocytes, infiltrating macrophages and local (myo)fibroblasts. It accumulates in dysfunctional adipose tissue, but can also be released into circulation. Higher levels of circulating endotrophin $(>6.3 \mathrm{ng} / \mathrm{ml}$ ) are associated with a better response to PPAR $\gamma$ agonists (i.e. TZDs), with fewer side effects. In contrast, individuals with lower endotrophin levels do not respond optimally to TZDs (in terms of fasting glucose and $\mathrm{HbA}_{1 \mathrm{c}}$ levels) and have an increased incidence of adverse side effects, such as peripheral oedema 
However, the detailed signalling pathways involved in these processes remain elusive.

The levels of endotrophin are upregulated in the adipose tissue of both diet-induced and genetically obese mouse models $[20,21]$. Importantly, we also recently established the upregulation of endotrophin in obese individuals [21]. Of note, we found that rather than a simple correlation with obesity, obese patients with insulin resistance displayed higher levels of endotrophin than obese individuals with normal insulin sensitivity. This suggests that endotrophin may serve as a useful biomarker for the level of metabolic fitness during the development of obesity [21].

The metabolic consequences of an excess of local endotrophin overexpression have been studied in different animal models [21]. The findings indicate that endotrophin is not only associated with an unfavourable microenvironment in obese adipose tissue, but that it is also a driving force associated with a pathology that ultimately leads to systemic insulin resistance and other metabolic disorders [21]. Specifically, we found that overexpression of endotrophin in obese adipose tissue stimulates fibrosis by upregulating ECM constituents. Moreover, through its potent chemoattractant activity, it leads to macrophage accumulation, resulting in an overall more enhanced proinflammatory microenvironment in adipose tissue. Both the pro-fibrotic and pro-inflammatory effects of endotrophin contribute to the metabolic dysfunction of adipose tissue, which in turn triggers systemic insulin resistance $[22,23]$.

How do the local pathological changes in adipose tissue, brought about by endotrophin, lead to systemic insulin resistance and generalised metabolic dysregulation? One of the contributory mechanisms may revolve around enhanced macrophage accumulation in the endotrophin-enriched adipose tissue. Macrophages are the major source of many of the circulating inflammatory factors in the obese state and are postulated to contribute to the development of insulin resistance [22]. In addition, the enhanced local fibrosis induced by endotrophin causes significant mechanical stress, limiting the ability of adipose tissue to effectively take up and esterify NEFAs. Adipose tissue with elevated endotrophin levels rapidly dysregulates circulating triacylglycerol and NEFA, and eventually causes hepatic steatosis [23]. Lipids thus start to accumulate ectopically in other tissues/organs, leading to a 'lipotoxic' environment that further promotes insulin resistance [24, 25]. We also observed that endotrophin-induced fibrosis leads to local dysfunction in brown adipose tissue, a process which may be a major mediator of the impaired ability to increase energy expenditure at the organismal level under conditions of elevated local levels of endotrophin [21]. Given the many adverse effects of endotrophin on the health and metabolic processes in adipose tissue, inhibition of endotrophin action via pharmacotherapy may constitute an effective strategy to reduce fibrosis and enhance metabolic flexibility. In rodent models, we have demonstrated that a neutralising antibody efficiently reduces endotrophin activity under high fat diet conditions. As a result, the antibody-treated mice exhibited a significant improvement in insulin sensitivity [21].

\section{Endotrophin as a biomarker for TZD responsiveness}

In this issue of Diabetologia, Karsdal and colleagues [26] provide a novel clinical assay in which they measure circulating endotrophin, a C-terminal cleavage fragment generated from the $\alpha 3$ chain of collagen VI. To date, very little is known about critical markers that may be available to identify drug response rates to TZDs. Specifically, the relationship between the response to TZDs and the level of ECM components is not well understood. In the present study [26], the authors divided participants into tertiles based on plasma endotrophin levels. They showed that serum endotrophin levels were positively associated with fasting glucose levels, as well as with HOMAIR, triacylglycerol, adiposity and indices of fatty liver. This is the first report associating the levels of endotrophin with other metabolic variables in humans. Indeed, these findings indicate that circulating endotrophin may be a useful additional indicator for the diagnosis of diabetes. In the present study, however, the most relevant observation relates to the fact that plasma endotrophin (most likely deriving from adipose tissue) can serve as a predictive biomarker for the response of diabetic individuals to TZD exposure [26]. In the upper two tertiles of endotrophin levels, the authors observe a more drastic reduction in fasting glucose and $\mathrm{HbA}_{1 \mathrm{c}}$ levels after TZD exposure than in individuals within the lowest tertile. This suggests that individuals with circulating endotrophin levels higher than $6.3 \mathrm{ng} / \mathrm{ml}$ are more responsive to TZDs (at least for the two compounds used here, pioglitazone and balaglitazone), while patients whose plasma endotrophin level is relatively lower at baseline do not respond well to these TZDs and may be better candidates for other glucose-lowering agents. In addition, in terms of adverse effects of the glitazone-based compounds, the incidence rate of peripheral oedema (considered a severe adverse effect [SAE]) was also related to endotrophin tertiles [26]. Thus endotrophin levels may serve as predictive indicators of at least some of the side effects of TZDs. However, the authors were unable to show a relationship between endotrophin levels and other SAEs, such as heart failure and cardiac ischaemia, because of the limited size of the cohort. Subsequent larger scale studies are required to provide further insights into possible associations between endotrophin levels and some of these other adverse events of TZDs. Nevertheless, Karsdal and colleagues [26] provide an exciting new biomarker to allow for a more customisable choice of insulin-sensitising regimen for optimal results in individual patients with type 2 diabetes. 


\section{How is endotrophin related to fibrosis?}

Collagen VI has been implicated in various fibrotic diseases, such as renal fibrosis, pulmonary fibrosis and hepatic fibrosis. In rodent models, collagen $\mathrm{VI}(\alpha 1)$ null mice (with a functional null phenotype for holo-collagen VI) are protected from myocardial infarction [27] and obesity-induced adipose tissue fibrosis $[23,28]$. In line with this, endotrophin showed potent profibrotic properties in the settings of both cancer [20,29] and diet-induced metabolic challenges [21]. This suggests that endotrophin accounts, at least in part, for the collagen VI-mediated fibrosis that is observed in various fibrotic diseases.

\section{The impact of TZDs on endotrophin in adipose tissue and the liver}

Fibrosis is a hallmark of chronic liver disease and is characterised by excessive accumulation and reorganisation of the ECM in the liver. Hepatic fibrosis is most successfully assessed using liver biopsies. When biopsy samples are unavailable for assessment, however, several biomarkers, including serum markers of liver function (i.e. alanine aminotransferase [ALT], aspartate aminotransferase [AST]), ECM synthesis (collagens, hyaluronan, glycoproteins), ECM degradation (matrix metalloproteinases [MMPs] and tissue inhibitor of metalloproteinases [TIMPs]), and fibrosis-related cytokines (TGF $\beta 1$, connective tissue growth factor [CTGF], plateletderived growth factor [PDGF], IL-4, IL-6) and adiponectin offer a valuable assessment of the degree of liver fibrosis present, although none of these biomarkers are conclusive. Collagen VI has also been suggested as a biomarker for hepatic fibrosis, showing a strong association from early to late hepatic fibrosis [30]. Consistent with this observation, we determined that endotrophin levels are significantly increased in liver biopsies from hepatic cirrhosis and hepatocellular carcinoma (HCC) patients, and our efforts are now towards determining the mechanisms of endotrophin-mediated hepatic fibrosis. Current data strongly supports the notion that endotrophin plays a crucial role in the pathogenesis of fibrotic disease beyond just being a biomarker of early fibrosis [21]. Obesity-induced dysfunctional adipose tissue contributes to hepatic lipotoxicity, leading to non-alcoholic fatty liver disease (NAFLD) and non-alcoholic steatohepatitis (NASH), both of which are frequent comorbidities in obese and diabetic patients. Diabetic patients who suffer from NAFLD or NASH can, under some circumstances, develop severe insulin resistance compared with those who are free of liver disease. These individuals may also move on to develop HCC. Several pharmacological agents have been tested in the context of NASH in type 2 diabetic populations; these include insulin sensitisers, glucagon-like peptide 1 (GLP-1) receptor antagonists, dipeptidyl peptidase IV (DPP-IV) inhibitors and sodium-glucose cotransporter 2 (SGLT2) inhibitors. A very recent clinical study by Cusi and colleagues revealed that long-term treatment with the TZD, pioglitazone, leads to an improvement of hepatic fibrosis in individuals with impaired glucose tolerance or type 2 diabetes who also have biopsyproven NASH [31]. Strikingly, TZDs treatment induced downregulation of COL6A3 mRNA levels in the adipose tissues of obese individuals, giving rise to the hypothesis that the antifibrotic effects of TZDs in diabetic patients with NASH could be partly mediated by suppression of endotrophin activity.

\section{TZDs and endotrophin in cancer and chemoresistance}

Endotrophin levels are significantly increased in human biopsies from various cancers, including cancers of the breast, colon and in pancreatic tumours. This suggests a role of endotrophin in cancer progression. This observation is strongly supported by experimental findings, using a mammary tumour mouse model (the mammary tumour virus-polyoma middle $\mathrm{T}$ antigen [MMTV-PyMT] transgenic mouse). Forced expression of endotrophin in the mammary epithelium in the background of the MMTV-PyMT mice dramatically increased both primary tumour size and pulmonary metastatic tumour growth. Endotrophin induced EMT and fibrosis through a TGF- $\beta$ dependent manner. Additionally, it stimulated tumour stromal expansion to promote chronic inflammation and angiogenesis via recruitment of immune cells and endothelial cells to the tumour microenvironment. Importantly, reconstitution of endotrophin into collagen VI null mice in the background of MMTV-PyMT mice completely bypassed the usual phenotype of reduced tumour growth in these animals that is caused by the functional absence of holocollagen VI microfilament formation, strongly supporting the notion that endotrophin is a strong tumour-promoting factor [20].

Resistance to chemotherapy is a major drawback of cancer treatment. One of the main causes of chemoresistance is the process of EMT that cancer cells undergo. In ovarian cancer cells, collagen $\mathrm{VI}(\alpha 3)$ is one of the most highly differentially expressed proteins in cisplatin- or oxaliplatin-resistant cancer cells compared with those that are sensitive to platinum compounds [32]. Our recent study revealed that endotrophin levels in mammary tumours were dramatically increased upon cisplatin treatment, leading to widespread cisplatin resistance resulting from stimulation of the EMT process in the cancer cells [19]. To overcome resistance to platinum-based chemotherapies, a combination of cisplatin and TZDs has been trialled and was found to confer greater effectiveness in cancer treatment as compared with platinum monotherapy. Furthermore, we found that the beneficial effects of TZDs 
on cisplatin therapies were due to the marked reduction of the endotrophin levels [19]. Consistent with this model, the inhibition of endotrophin activity via use of therapeutic monoclonal antibodies conferred cisplatin sensitivity in the mice. Therefore, we speculate that high levels of endotrophin in tumours are responsible for the reported beneficial effects of TZDs in chemotherapies [33, 34].

TZDs, including troglitazone, rosiglitazone, pioglitazone and ciglitazone showed an anti-tumour growth effect in preclinical studies, translated to beneficial effects in some clinical trials $[33,34]$. We have now learned that TZD action in tumours is highly complex and is not necessarily correlated with PPAR $\gamma$ activation status. TZDs have been found to have a beneficial effect in prostate cancer and in gliomas, but the efficacy of TZD monotherapy in cancer is relatively low. However, a meta-analysis of data from clinical diabetes studies and also in vitro studies revealed that TZDs could be considered as chemosensitising agents in colon, breast and lung cancers. Most of the in vitro studies showed anti-tumour effects of TZDs when combined with chemotherapy agents [35, 36]. In humans, a beneficial effect was found in a subset of tumours, such as thyroid cancer, soft tissue sarcoma and melanoma, with combined treatment of TZDs and conventional chemotherapy drugs. The mechanisms underlying the effects of TZD on tumours are still elusive because of the complex responses elicited by TZDs in different cells and at different stages of tumour progression. So why do TZDs trigger such a complex response in different cancer types? A differential complement of transcriptional co-repressors and coactivators in different cell types is likely to contribute. Also, what factors determine the efficacy of TZDs on tumours? This is unknown but the availability of biomarkers such as endotrophin may be helpful for adjustment of the therapeutic strategy used on a case-by-case basis.

\section{Remaining questions}

While the crucial functions of endotrophin in metabolic dysregulation and tumour growth have been highlighted, our insights into the detailed mechanisms by which endotrophin exerts its actions are currently limited. Several questions remain to be addressed in future studies; first, what is the stepwise process that leads to the cleavage of endotrophin from its parental collagen $\operatorname{VI}(\alpha 3)$ molecule? This includes the time course of obesity development, the location of the cell compartment, the involvement of specific endopeptidase(s) and the exact cleavage site(s) in the parental collagen $\operatorname{VI}(\alpha 3)$ chain. Second, which cell surface receptors does endotrophin bind to activate downstream events, and what are the signal transduction pathways that endotrophin induces, leading to the pathological reactions in both tumours and adipose tissue? Finally, what levels of endotrophin accumulation occur in other metabolically active tissues/organs, such as the muscle, the liver and the heart, and what are the functions of endotrophin in these tissues/organs? Answering these questions will help us better understand how endotrophin exerts its functions in metabolically active sites, such as adipose tissue, muscles and tumours, hence offering us new therapeutic strategies to target this novel adipokine for the treatment of metabolic diseases and cancer. Most importantly, research in this area will shed light onto why endotrophin is such an excellent predictive biomarker for the overall glucoselowering response to TZD treatment, as demonstrated by Karsdal and colleagues [26].

Funding The authors are supported by the National Institute of Health (R01-DK55758, R01-DK099110 and P01-DK088761) as well as a grant from the Cancer Prevention and Research Institute of Texas (CPRIT RP140412) awarded to PES. JP is also supported by a Korea Health Technology Research and Development Project grant through the Korea Health Industry Development Institute (KHIDI), funded by the Ministry of Health and Welfare, Republic of Korea (HI14C1277) and a Basic Science Research Program grant through the National Research Foundation of Korea (NRF) funded by the Ministry of Education (NRF2014R1A1A2054914). KS is supported by a Pilot award from the Clinical and Translational Science Center (CCTS) at the University of Texas Health Science Center at Houston (UL1 TR000371). MK is supported by the Priority Research Centers Program through the NRF, funded by the Ministry of Education, Science and Technology (2010-0020224).

Duality of interest PES is the recipient of a sponsored research agreement with Panamab, a therapeutic antibody company in Houston, TX, USA.

Contribution statement All authors were responsible for drafting the article and revising it critically for important intellectual content. All authors approved the final version to be published.

\section{References}

1. Spiegelman BM (1998) PPAR-gamma: adipogenic regulator and thiazolidinedione receptor. Diabetes 47:507-514

2. Kliewer SA, Sundseth SS, Jones SA et al (1997) Fatty acids and eicosanoids regulate gene expression through direct interactions with peroxisome proliferator-activated receptors alpha and gamma. Proc Natl Acad Sci U S A 94:4318-4323

3. Lehmann JM, Moore LB, Smith-Oliver TA, Wilkison WO, Willson TM, Kliewer SA (1995) An antidiabetic thiazolidinedione is a high affinity ligand for peroxisome proliferator-activated receptor $\gamma$ (PPAR $\gamma$ ). J Biol Chem 270:12953-12956

4. Nissen SE, Wolski K (2007) Effect of rosiglitazone on the risk of myocardial infarction and death from cardiovascular causes. N Engl J Med 356:2457-2471

5. He J, Xu C, Kuang J et al (2015) Thiazolidinediones attenuate lipolysis and ameliorate dexamethasone-induced insulin resistance. Metabolism 64:826-836

6. Mayerson AB, Hundal RS, Dufour S et al (2002) The effects of rosiglitazone on insulin sensitivity, lipolysis, and hepatic and 
skeletal muscle triglyceride content in patients with type 2 diabetes. Diabetes 51:797-802

7. Chung SS, Kim M, Youn BS et al (2009) Glutathione peroxidase 3 mediates the antioxidant effect of peroxisome proliferator-activated receptor gamma in human skeletal muscle cells. Mol Cell Biol 29: 20-30

8. Hammarstedt A, Andersson CX, Rotter Sopasakis V, Smith U (2005) The effect of PPARgamma ligands on the adipose tissue in insulin resistance. Prostaglandins Leukot Essent Fatty Acids 73:65-75

9. Berg AH, Combs TP, Du X, Brownlee M, Scherer PE (2001) The adipocyte-secreted protein Acrp30 enhances hepatic insulin action. Nat Med 7:947-953

10. Boden G, Homko C, Mozzoli M, Showe LC, Nichols C, Cheung P (2005) Thiazolidinediones upregulate fatty acid uptake and oxidation in adipose tissue of diabetic patients. Diabetes 54:880-885

11. Hu Y, Liu HB, Simpson RW, Dear AE (2011) PPARgammaindependent thiazolidinedione-mediated inhibition of NUR77 expression in vascular endothelial cells. J Endocrinol 208:R1-R7

12. Suter SL, Nolan JJ, Wallace P, Gumbiner B, Olefsky JM (1992) Metabolic effects of new oral hypoglycemic agent CS-045 in NIDDM subjects. Diabetes Care 15:193-203

13. Igarashi M, Jimbu Y, Kimura M, Hirata A, Yamaguchi H, Tominaga M (2007) Effect of pioglitazone on atherogenic outcomes in type 2 diabetic patients: a comparison of responders and non-responders. Diabetes Res Clin Pract 77:389-398

14. Satoh N, Ogawa Y, Usui T et al (2003) Antiatherogenic effect of pioglitazone in type 2 diabetic patients irrespective of the responsiveness to its antidiabetic effect. Diabetes Care 26:2493-2499

15. Nawrocki AR, Rajala MW, Tomas E et al (2006) Mice lacking adiponectin show decreased hepatic insulin sensitivity and reduced responsiveness to peroxisome proliferator-activated receptor gamma agonists. J Biol Chem 281:2654-2660

16. Sears DD, Hsiao G, Hsiao A et al (2009) Mechanisms of human insulin resistance and thiazolidinedione-mediated insulin sensitization. Proc Natl Acad Sci U S A 106:18745-18750

17. Gara SK, Grumati P, Urciuolo A et al (2008) Three novel collagen VI chains with high homology to the alpha3 chain. J Biol Chem 283:10658-10670

18. Colombatti A, Mucignat MT, Bonaldo P (1995) Secretion and matrix assembly of recombinant type VI collagen. J Biol Chem 270: 13105-13111

19. Park J, Morley TS, Scherer PE (2013) Inhibition of endotrophin, a cleavage product of collagen VI, confers cisplatin sensitivity to tumours. EMBO Mol Med 5:935-948

20. Park J, Scherer PE (2012) Adipocyte-derived endotrophin promotes malignant tumor progression. J Clin Invest 122:4243-4256
21. Sun K, Park J, Gupta OT et al (2014) Endotrophin triggers adipose tissue fibrosis and metabolic dysfunction. Nat Commun 5:3485

22. Sun K, Kusminski CM, Scherer PE (2011) Adipose tissue remodeling and obesity. J Clin Invest 121:2094-2101

23. Sun K, Tordjman J, Clement K, Scherer PE (2013) Fibrosis and adipose tissue dysfunction. Cell Metab 18:470-477

24. Kusminski CM, Shetty S, Orci L, Unger RH, Scherer PE (2009) Diabetes and apoptosis: lipotoxicity. Apoptosis 14:1484-1495

25. Unger RH, Scherer PE (2010) Gluttony, sloth and the metabolic syndrome: a roadmap to lipotoxicity. Trends Endocrinol Metab $21: 345-352$

26. Karsdal MA, Henriksen K, Genovese F et al (2016) Serum endotrophin identifies optimal responders to PPAR $\gamma$ agonists in type 2 diabetes. Diabetologia. doi:10.1007/s00125-016-4094-1

27. Luther DJ, Thodeti CK, Shamhart PE et al (2012) Absence of type VI collagen paradoxically improves cardiac function, structure, and remodeling after myocardial infarction. Circ Res 110:851-856

28. Khan T, Muise ES, Iyengar P et al (2009) Metabolic dysregulation and adipose tissue fibrosis: role of collagen VI. Mol Cell Biol 29: 1575-1591

29. Park J, Scherer PE (2013) Endotrophin in the tumor stroma: a new therapeutic target for breast cancer? Expert Rev Anticancer Ther 13: $111-113$

30. Stickel F, Urbaschek R, Schuppan D et al (2001) Serum collagen type VI and XIV and hyaluronic acid as early indicators for altered connective tissue turnover in alcoholic liver disease. Dig Dis Sci 46: 2025-2032

31. Cusi K, Orsak B, Bril F et al (2016) Long-term pioglitazone treatment for patients with nonalcoholic steatohepatitis and prediabetes or type 2 diabetes mellitus: a randomized trial. Ann Intern Med 165: 305-315

32. Sherman-Baust CA, Weeraratna AT, Rangel LB et al (2003) Remodeling of the extracellular matrix through overexpression of collagen VI contributes to cisplatin resistance in ovarian cancer cells. Cancer Cell 3:377-386

33. Blanquicett C, Roman J, Hart CM (2008) Thiazolidinediones as anti-cancer agents. Cancer Ther 6:25-34

34. Palakurthi SS, Aktas H, Grubissich LM, Mortensen RM, Halperin JA (2001) Anticancer effects of thiazolidinediones are independent of peroxisome proliferator-activated receptor gamma and mediated by inhibition of translation initiation. Cancer Res 61:6213-6218

35. Girnun GD, Naseri E, Vafai SB et al (2007) Synergy between PPARgamma ligands and platinum-based drugs in cancer. Cancer Cell 11:395-406

36. Tikoo K, Kumar P, Gupta J (2009) Rosiglitazone synergizes anticancer activity of cisplatin and reduces its nephrotoxicity in 7, 12dimethyl benz $\{a\}$ anthracene (DMBA) induced breast cancer rats. BMC Cancer 9:107 